\title{
Desempenho motor e estado nutricional de escolares com e sem transtorno do desenvolvimento da coordenação
}

\author{
Motor performance and nutritional status of schoolchildren \\ with and without developmental coordination disorder
}

\author{
Talita Barbosa Miranda \\ Thaís Silva Beltrame \\ Fernando Luiz Cardoso
}

1. Universidade do Estado de Santa Catarina. Programa de Pós-Graduação em Ciências do Movimento Humano; Laboratório de Distúrbios da Aprendizagem e do Desenvolvimento. Florianópolis, SC, Brasil.

Recebido em 20/05/10 Revisado em 03/06/10 Aprovado em 28/07/10
Resumo - O objetivo desse estudo foi investigar o desempenho motor e o estado nutricional de escolares de 7 a 10 anos de idade, com e sem Transtorno do Desenvolvimento da Coordenação (TDC). Participaram do estudo, 380 escolares de escolas públicas de Florianópolis -SC -Brasil. Para a identificação de dificuldades motoras, foi utilizado o Movement Assessment Battery for Children (MABC-2) e para a avaliação do estado nutricional, foram realizadas medidas antropométricas de peso e altura e cálculo do IMC. Os resultados demonstraram que 6,1\% $(n=23)$ apresentaram TDC, sendo 18 meninos e 5 meninas. No grupo com TDC, observou-se diferença significativa entre as habilidades de destreza manual e habilidades de lançar/receber e entre as habilidades de lançar/receber e equilíbrio. Quanto ao estado nutricional, $85,5 \%$ dos escolares, em geral, apresentaram eutrofia e 12,9\% apresentaram sobrepeso/obesidade. No grupo com TDC, 91,3\% obtiveram eutrofia e 8,7\% apresentaram sobrepeso. Não houve associação entre o estado nutricional e desempenho motor entre os dois grupos, o que nos leva a perceber que o estado nutricional não influenciou o desempenho motor dos escolares avaliados.

Palavras-chave: Transtornos das habilidades motoras; Estado nutricional; Destreza motora.

Abstract - The objective of this study was to investigate the motor performance and nutritional status of schoolchildren aged 7 to 10 years with developmental coordination disorder (DCD). A total of 380 children from public schools in Florianópolis, SC, Brazil, participated in the study. The Movement Assessment Battery for Children (MABC-2) was used for the identification of motor difficulties. Weight and height were measured and the body mass index (BMI) was calculated for the evaluation of nutritional status. The results showed that $6.1 \%(n=23)$ of the students presented signs of DCD, including 18 boys and 5 girls. In the group with DCD, a significant difference was observed between manual dexterity skills and aiming/catching skills and between aiming/catching skills and balance. Regarding nutritional status, 85.5\% of all students were eutrophic and $12.9 \%$ were obese. In the group with DCD, 91.3\% of the students were eutrophic and $8.7 \%$ were obese. There was no association between nutritional status and motor performance in the two groups, indicating that nutritional status did not influence motor performance in the schoolchildren studied.

Key words: Motor skills disorders; Nutritional status; Motor skills. 


\section{INTRODUÇÃO}

No início da infância, a criança começa a obter o controle de suas habilidades motoras fundamentais, possuindo um potencial de desenvolvimento que a orienta ao estágio maduro por volta da idade de seis anos. Em torno dos sete anos de idade até, aproximadamente, aos dez, as crianças entram no estágio transitório de habilidades motoras, quando passam a combinar e a aplicar as habilidades fundamentais ao desempenho de habilidades especializadas, podendo este desempenho ser influenciado por fatores extrínsecos e intrínsecos ao indivíduo, como as condições sociais e estado nutricional ${ }^{1}$. Entretanto, para crianças que apresentam dificuldades motoras, o movimento pode se constituir um grande desafio, de maneira que determinadas tarefas típicas da infância se tornam bastante difíceis² .

Crianças com dificuldades motoras receberam diversos termos ao longo do tempo, tais como clumsy ou clumsiness (desajeitado ou desajeitamento), disfunção sensório-motora desenvolvimental, dispraxia desenvolvimental, Dificuldade Cerebral Mínima, Dificuldade Neurológica Mínima e incapacidade ${ }^{3}$. Na década de 1980 , este caso foi reconhecido pela Organização Mundial de Saúde - OMS e pela Associação de Psiquiatria Americana - APA, recebendo o nome de Developmental Coordination Disorder (DCD), traduzido para o português como Transtorno do Desenvolvimento da Coordenação (TDC) ${ }^{4}$. Dentre as características das crianças com TDC estão o desajeitamento em seus movimentos, a dificuldade com habilidades motoras grossas e finas, o atraso no desenvolvimento de algumas habilidades motoras como andar de bicicleta, receber bola, manejar faca e garfo e dificuldades na escrita 5 .

A porcentagem do TDC está entre 5\% a 15\% das crianças em idade escolar, sendo identificadas, geralmente, a partir dos quatro anos de idade ${ }^{6}$. Estudos demonstram as consequências que as dificuldades motoras podem trazer ao indivíduo, tanto no aspecto físico por evitar atividades físicas, como a hipertensão arterial, alto nível de colesterol, sobrepeso e obesidade, quanto no social, emocional e cognitivo, incluindo nível de ansiedade elevado, baixa autoestima e exclusão social ${ }^{6,7}$.

Nas últimas décadas, estudiosos de diversos países têm voltado a atenção para crianças com TDC $^{8,9}$. No Brasil, estudos sobre este transtorno cresceu nos últimos anos, abordando aspectos relacionados à prevalência, intervenções e estudos de levantamento bibliográfico ${ }^{10,11}$. Porém, apesar de que algumas pesquisas em escolas brasileiras tenham apontado crianças com TDC, pouco se sabe a respeito do desenvolvimento motor em termos de desempenho motor e estado nutricional das mesmas, sendo escassa a literatura que aborda o desempenho motor e estado nutricional de crianças com TDC. Acredita-se que quanto mais cedo for diagnosticado o TDC em escolares e poder verificar o seu desempenho motor e estado nutricional, mais cedo poderão ser feitas as intervenções necessárias para que eles tenham um desenvolvimento mais saudável e melhor qualidade de vida.

Portanto, esse estudo buscou investigar o desempenho motor e o estado nutricional de escolares de 7 a 10 anos de idade, comparando os participantes com e sem Transtorno do Desenvolvimento da Coordenação.

\section{PROCEDIMENTOS METODOLÓGICOS}

\section{Local do estudo e amostra}

Esta pesquisa foi realizada na parte continental do município de Florianópolis-SC, na região Sul do Brasil. Essa parte do município possui treze escolas públicas para os anos iniciais do ensino fundamental, totalizando 3268 alunos matriculados no ano letivo de $2009^{12}$. Para a execução do estudo, foi entregue o projeto para os diretores e/ ou coordenadores pedagógicos das escolas, sendo explicitado verbalmente os objetivos e a dinâmica da pesquisa. Em seguida, os alunos receberam os Termos de Consentimento Livre e Esclarecido, para que fossem assinados pelos pais ou responsáveis, autorizando a participação da criança no estudo.

A seleção da amostra foi realizada em duas etapas. Na primeira, foram selecionadas as escolas, perfazendo um total de seis e, na segunda, foram selecionados os alunos participantes. Em relação à seleção dos participantes, quatro escolas permitiram a participação de todos os alunos do $1^{\circ}$ ao $5^{\circ}$ ano, que tinham idade correspondente ao estudo, e todos que estavam no dia da entrega receberam o termo ( $\mathrm{n}=540)$; uma escola liberou apenas os alunos que faziam parte de um projeto social ali desenvolvido $(\mathrm{n}=83)$. Em outra escola, somente duas turmas de $2^{\circ}$ ano e uma de $3^{\circ}$ foram liberadas para participar da pesquisa, sendo que todos os alunos dessas turmas receberam os termos $(\mathrm{n}=87)$. No total, 710 termos de consentimento foram entregues às crianças para levarem aos seus pais.

Para inclusão das crianças na amostra, foram estabelecidos quatro critérios: a) comparecimento à escola no dia das avaliações; b) possuir o termo de 
consentimento assinado por um dos responsáveis da criança; c) não recusar em participar da coleta de dados e (d) não possuir algum problema físico e/ou condição médica geral que o impedisse temporária ou definitivamente de realizar a coleta, sendo identificado por observação dos avaliadores ou por indicação do professor e/ou da direção da escola e/ou dos responsáveis pela criança. A amostra final foi composta por 380 crianças de ambos os sexos, com idade média de 8,5 anos $( \pm 1,1)$, com um erro amostral tolerado de $5 \%$.

O projeto foi aprovado pelo Comitê de Ética em Pesquisa em Seres Humanos (CEP) da UDESC por cumprir os termos da resolução 169/96 do Conselho Nacional de Saúde.

\section{Instrumentos de avaliação}

Para avaliar o desempenho motor, foi utilizado o Movement Assessment Battery for Children MABC-2 para a faixa etária 2, que compreende as idades de 7 a 10 anos $^{6}$. O MABC-2 tem se tornado o teste mais usado para operacionalizar o critério A do DSM-IV, o qual diz que o TDC pode manifestar-se por atrasos marcantes em alcançar marcos motores, propensão a deixar cair objetos, desajeitamento, fraco desempenho nos esportes e/ ou caligrafia insatisfatória ${ }^{6}$.

O MABC-2 é composto por oito tarefas que envolvem habilidades de destreza manual, habilidades de lançar/receber e habilidades de equilíbrio estático e dinâmico, medidos pelo tempo em segundos, número de erros e número de acertos, dependendo da tarefa. $O$ teste categoriza as crianças de acordo com o grau de dificuldade motora. Os escores padrão variam de 1 a 19 e para cada valor existe o percentil correspondente, que varia de $0,1 \%$ a $99,9 \%$. Os valores são distribuídos de forma que igual ou abaixo do $5^{\circ}$ percentil indica uma significativa dificuldade no movimento; entre o $6^{\circ}$ e o $15^{\circ}$ percentil sugere que a criança tem risco de dificuldade e, acima ou igual ao $16^{\circ}$ percentil demonstra que a criança não possui nenhuma dificuldade.

Para verificar o estado nutricional, foram realizadas medidas antropométricas de peso, utilizando-se uma balança digital portátil, modelo Tanita, com capacidade para $150 \mathrm{~kg}$ e graduação em 100 g e, para a medida da estatura, foi utilizado um estadiômetro extensível, com escala em milímetros. Com essas medidas, foi feito o cálculo do índice de massa corporal (IMC). A classificação do IMC foi realizada de acordo com os dados de referência para crianças e adolescentes de 5 a 19 anos de idade da Organização Mundial de Saúde ${ }^{13}$. Neste estudo, utilizaram-se os valores individuais dos escores-Z de IMC para idade.

\section{Coleta de dados}

A coleta de dados aconteceu na própria escola, em ambientes adequados (salas de aula, ginásio ou pátio) cedida pela direção escolar. $\mathrm{O}$ MABC-2 foi aplicado de maneira individual seguindo o seu protocolo. As tarefas eram explicitadas e demonstradas aos alunos, sendo tirada qualquer dúvida que eles tivessem. As crianças tinham um número específico de treino de acordo com cada tarefa, como se estivessem simulando o que iriam fazer nas práticas.

Para a aferição dos dados antropométricos, a medida de massa corporal foi realizada por meio de balança digital, colocada em superfície plana, zerada a cada pesagem. $\mathrm{O}$ indivíduo permaneceu em posição ortostática com os membros superiores estendidos ao longo do corpo, com os pés descalços e roupas leves. A medida da estatura foi realizada em centímetros, por meio de estadiômetro fixado em uma parede lisa, a 90 graus em relação ao piso.

\section{Análise estatística}

Para análise dos dados, foi utilizado o programa SPSS 15.0 for Windows. Para verificar a incidência de TDC entre os escolares bem como o estado nutricional dos mesmos, foi utilizada estatística descritiva de distribuição de frequência. Para verificar as associações de incidência de TDC entre os sexos e faixas etárias, foi utilizado o teste Qui-quadrado $\left(\mathrm{X}^{2}\right)$, bem como para fazer associações do estado nutricional entre os sexos e faixas etárias e estado nutricional e classificação motora. Para comparações do desempenho motor total entre os sexos e faixas etárias, foi utilizado o Teste $\mathrm{T}$ independente e Análise de Variância (ANOVA one-way) respectivamente, bem como para a comparação do estado nutricional dos escolares com e sem TDC entre os sexos e faixas etárias.

Para verificar a diferença no desempenho motor, de acordo com cada habilidade, do grupo que apresentou TDC, foi utilizada a ANOVA para medidas repetidas. Para comparar o desempenho motor por habilidades entre os sexos, faixas etárias e por classificação no MABC-2, utilizou-se a análise multivariada MANOVA.

Para todas as análises estatísticas, foi adotado o nível de significância de 5\%, o nível de alfa definido como 0,05 e o intervalo de confiança de 95\%.

\section{RESULTADOS}

\section{Desempenho motor}

Ao observar a frequência de escolares com dificuldade no movimento, sugestivo de TDC, verificou-se 
que o sexo masculino foi o que mais apresentou esta dificuldade. A tabela 1 apresenta a frequência de TDC por sexo e idade.

Observando os dados gerais, foi encontrada uma associação significativa entre classificação motora e sexo $\left(X^{2}=9,07 ; p=0,011\right)$. Já entre as idades, não foi encontrada associação significativa $\left(X^{2}=10,17 ; p=0,117\right)$.

Quando comparadas as médias, entre os sexos, de cada habilidade (tabela 2), verificou-se que houve diferença significativa entre meninos e meninas nas habilidades de destreza manual, nas habilidades de lançar e receber e nas habilidades de equilíbrio.

Analisando a diferença entre as faixas etárias por habilidade (destreza manual, lançar e receber, equilíbrio estático e dinâmico), utilizando para esse fim a pontuação total de cada habilidade, percebeu-se que não houve diferença significativa entre faixa etária e habilidade de destreza manual e entre a faixa etária e habilidade de lançar e receber, porém entre a faixa etária e habilidades de equi- líbrio estático e dinâmico, percebeu-se diferença significativa (tabela 3). Verificando as múltiplas comparações de Bonferroni, observou-se que essa diferença significativa ocorreu entre as idades de 7 e 9 anos $(p=0,031)$ e entre 7 e 10 anos $(p=0,010)$.

No grupo dos escolares que apresentaram TDC, as meninas apresentaram maior pontuação que os meninos nas habilidades de destreza manual, porém essa diferença não foi significativa $(p=0,790)$; nas habilidades de lançar e receber e nas habilidades de equilíbrio, os meninos obtiveram uma média de pontuação mais alta, apesar dessa diferença não ter apresentado significância estatística ( $p=0,796$ e $\mathrm{p}=0,716$ respectivamente) (tabela 4 ).

Verificando a diferença no desempenho motor, conforme cada habilidade do grupo que apresentou TDC, observou-se diferença significativa entre as médias das habilidades $\left(\mathrm{F}_{(1,66)}=7,391 ; \mathrm{p}=0,003\right)$. Comparações pareadas de Bonferroni demonstraram que entre as habilidades de destreza manual e equilíbrio não houve diferença significativa $(\mathrm{p}=1,000)$, porém

Tabela 1. Frequência da graduação das dificuldades motoras de acordo com sexo e idade.

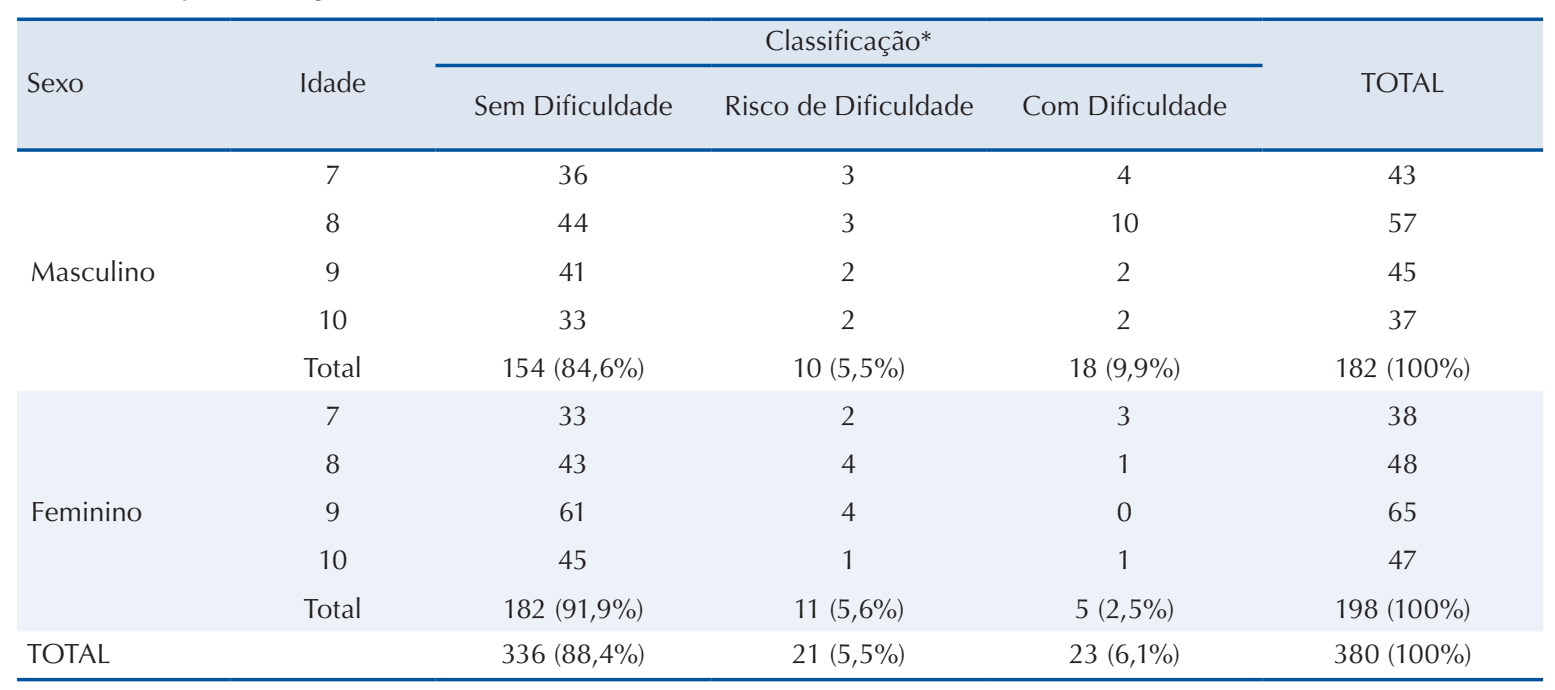

*Sem dificuldade: acima do $15^{\circ}$ percentil; Risco de dificuldade: entre o $5^{\circ}$ e o $15^{\circ}$ percentil;

Dificuldade significativa: abaixo do $5^{\circ}$ percentil.

Tabela 2. Desempenho motor por habilidade entre os sexos.

\begin{tabular}{|c|c|c|c|c|c|c|c|c|c|}
\hline \multirow{2}{*}{$\begin{array}{l}\text { Tipos de } \\
\text { habilidades }\end{array}$} & \multirow{2}{*}{ Sexo } & \multirow{2}{*}{$\mathrm{n}$} & \multirow{2}{*}{ Média } & \multirow{2}{*}{ DP } & \multicolumn{2}{|c|}{ Intervalo de confiança 95\% } & \multirow{2}{*}{$\mathrm{F}(\mathrm{gl})$} & \multirow{2}{*}{$\mathrm{p}$} & \multirow{2}{*}{$\eta^{2}$} \\
\hline & & & & & Inferior & Superior & & & \\
\hline Destreza Manual & Feminino & 198 & 26,78 & 5,17 & 26,04 & 27,52 & $8,460(1)$ & 0,004 & $2,2 \%$ \\
\hline Lançar e Receber & Masculino & 182 & 19,32 & 4,87 & 18,68 & 19,96 & $23,521(1)$ & $<0,001$ & $5,9 \%$ \\
\hline \multirow{2}{*}{ Equilíbrio } & Masculino & 182 & 30,45 & 6,30 & 29,63 & 31,26 & \multirow{2}{*}{$11,941(1)$} & \multirow{2}{*}{0,001} & \multirow{2}{*}{$3,1 \%$} \\
\hline & Feminino & 198 & 32,44 & 4,90 & 31,65 & 33,22 & & & \\
\hline
\end{tabular}

$\mathrm{N}=$ amostra; $\mathrm{DP}=$ desvio padrão; $\eta^{2}=$ tamanho do efeito 
Tabela 3. Desempenho motor por habilidade entre as idades.

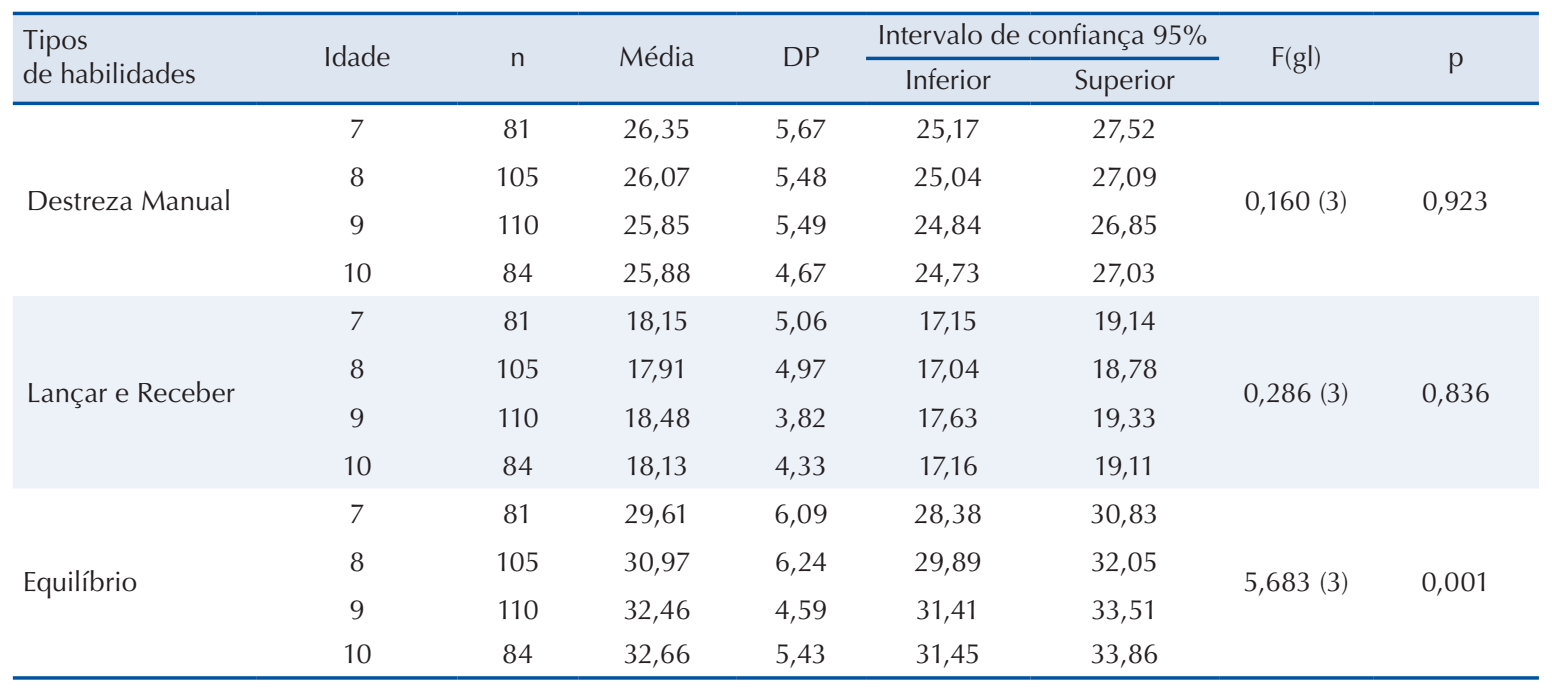

$\mathrm{n}=$ amostra; DP= desvio padrão.

Tabela 4. Medidas descritivas dos escolares com TDC divididas por habilidades de acordo com sexo e idade.

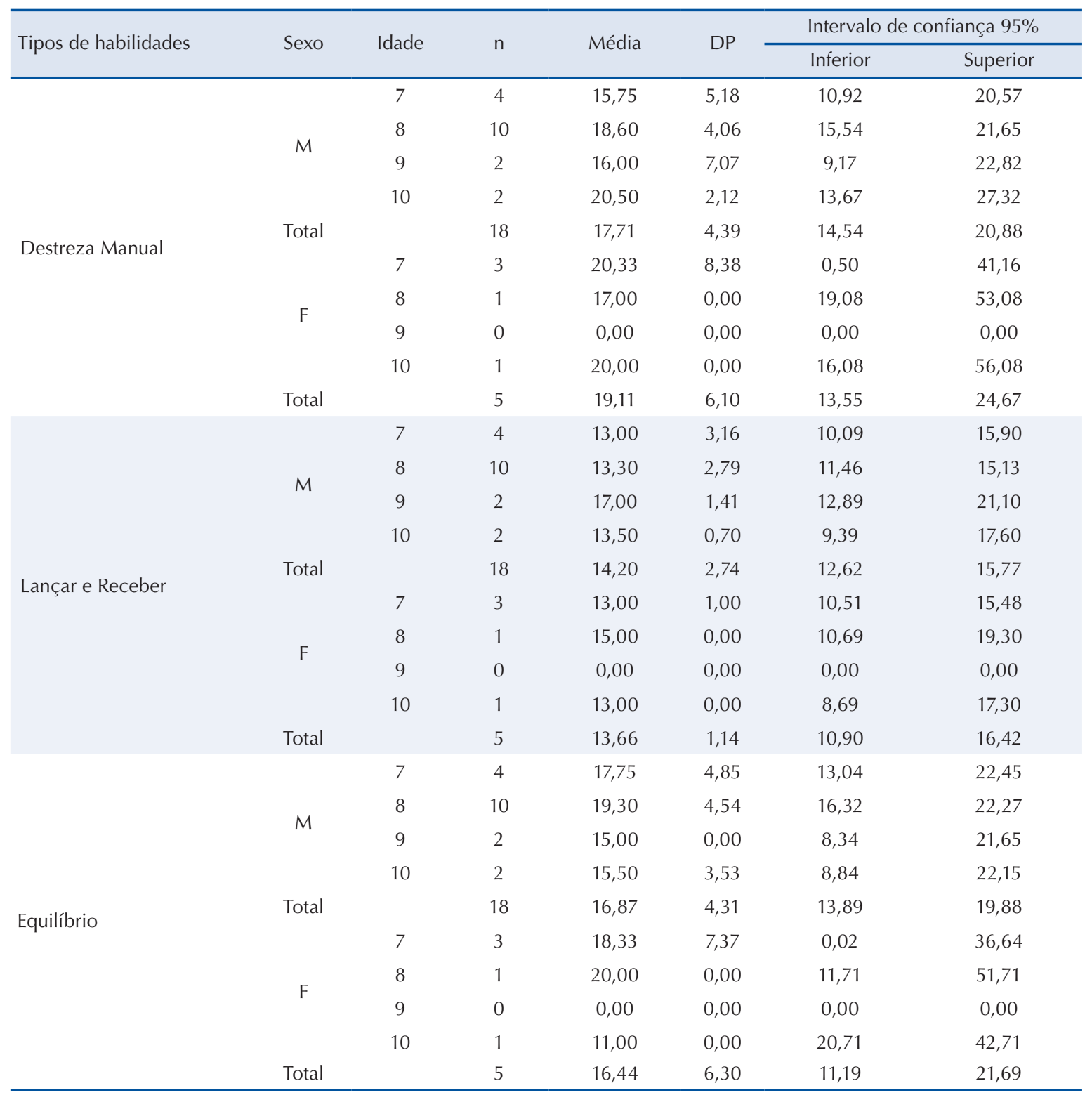

$M=$ masculino; $F=$ feminino. 
houve diferença significativa entre as habilidades de destreza manual e habilidades de lançar e receber $(p=0,003)$ e entre as habilidades de lançar e receber e equilíbrio $(p=0,003)$. Esses dados sugerem que os escolares com TDC apresentaram resultados inferiores nas habilidades de lançar e receber.

\section{Estado nutricional}

Analisando o estado nutricional dos escolares em geral, notou-se que a maioria apresentou eutrofia, assim também dentre os alunos com TDC (tabela 5).

Verificando o estado nutricional por sexo, de acordo com a idade e classificação motora, os resultados mostraram que os dois alunos com TDC, que apresentaram sobrepeso, são do sexo masculino, sendo ambos da idade de 8 anos. Já os dois alunos com risco de dificuldade que apresentaram sobrepeso, 1 é do sexo masculino, da idade de 9 anos, e 1 é do sexo feminino, também da idade de 9 anos.

Quanto à associação entre classificação motora e estado nutricional dividida por sexo, constatou-se que não houve associação entre estado nutricional e classificação motora no sexo masculino $\left(X^{2}=2,63\right.$; $\mathrm{p}=0,989$ ) e estado nutricional e classificação motora no sexo feminino $\left(X^{2}=1,46 ; p=0,993\right)$, o que neutraliza qualquer influência do estado nutricional sobre a classificação motora.

\section{DISCUSSÃO}

O presente estudo teve como objetivo investigar o desempenho motor e o estado nutricional de escolares com e sem TDC. Raríssimos são os estudos que envolvem essa temática, em se tratando de crianças com transtornos motores.

A incidência de crianças com atraso motor sugestivo de TDC encontrada no estudo equivaleu ao valor reportado pela $\mathrm{APA}^{4}$, que indica uma prevalência em torno de $6 \%$ em crianças de 5 a 11 anos de idade. A frequência dessa desordem tem sido variada entre os países. Alguns autores definem uma porcentagem de $6 \%$ a $13 \%$ ou entre $5 \%$ a $15 \%$ de crianças em idade escolar que apresentam dificuldade motora severa ${ }^{14}$ e em torno de $10 \%$ estão incluídas na categoria de risco, ou dificuldade motora moderada ${ }^{6,15}$.

O sexo masculino apresentou maior número de casos de TDC do que o sexo feminino, mostrando uma relação de quase 4:1. Esses dados foram equivalentes aos encontrados em estudos internacionais, que apontam uma relação que varia de 3 a 4 meninos para 1 menina $(4: 1)$ a 7 meninos para 1 menina $(7: 1)^{16-18}$

Quanto ao desempenho geral no teste MABC2, o sexo feminino apresentou melhor resultado do que o sexo masculino, embora essa diferença não tenha sido significativa. Quando analisado o desempenho por habilidade, nas habilidades de destreza manual e de equilíbrio as meninas apresentaram melhores resultados, porém, nas habilidades de lançar e receber, os meninos se apresentaram melhores. Entre os escolares que apresentaram TDC, no total, eles apresentaram escores mais baixos nas habilidades de lançar e receber. Quando verificado o desempenho motor entre os sexos, apesar de não ter havido nenhuma diferença significativa, as meninas apresentaram uma pontuação mais alta do que os meninos nas habilidades de destreza manual, entretanto, nas habilidades de lançar e receber e de equilíbrio, os meninos obtiveram maior pontuação. Esses achados corroboram vários estudos que argumentam que as meninas são melhores em habilidades de agilidade, de destreza manual e em habilidades de equilíbrio estático e dinâmico, enquanto os meninos são melhores em habilidades com bola, em saltos verticais e horizontais e em corrida de velocidade ${ }^{19}$.

Quanto ao estado nutricional, no geral, a maioria dos escolares apresentou eutrofia. No entanto, a taxa de sobrepeso/obesidade dos escolares do presente estudo deve ser levada em conta $(\mathrm{SO}+\mathrm{OB}+\mathrm{OG}=12,9 \%$ escolares $)$, já que o excesso de peso traz sérias consequências ao indivíduo e relaciona-se com várias comorbidades, como hipertensão arterial, diabetes mellitus, enfermidades respiratórias, problemas ortopédicos, acidente

Tabela 5. Frequência do estado nutricional dos escolares com e sem TDC distribuída por sexo e idade .

\begin{tabular}{|c|c|c|c|c|c|c|c|c|c|c|c|c|c|}
\hline \multirow{2}{*}{ Classificação } & \multirow{2}{*}{$\mathrm{n}$} & \multicolumn{12}{|c|}{ Estado Nutricional } \\
\hline & & MA & $\%$ & M & $\%$ & $\mathrm{E}$ & $\%$ & $S$ & $\%$ & $\mathrm{OB}$ & $\%$ & OG & $\%$ \\
\hline Sem dificuldade & 336 & 2 & 0,6 & 4 & 1,2 & 285 & 84,8 & 31 & 9,2 & 10 & 3,0 & 4 & 1,2 \\
\hline Risco de dificuldade & 21 & 0 & 0 & 0 & 0 & 19 & 90,5 & 2 & 9,5 & 0 & 0 & 0 & 0 \\
\hline Com dificuldade & 23 & 0 & 0 & 0 & 0 & 21 & 91,3 & 2 & 8,7 & 0 & 0 & 0 & 0 \\
\hline Total & 380 & 2 & 0,6 & 4 & 1,2 & 325 & 85,5 & 35 & 9,2 & 10 & 2,6 & 4 & 1,1 \\
\hline
\end{tabular}

*MA- magreza acentuada; M- magreza; E- eutrofia; S- sobrepeso; OB- obesidade; OG- obesidade grave (dados OMS/ SISVAN, 2007) 
vascular cerebral, doenças coronarianas, problemas psicológicos, diminuição da autoestima entre outros $^{20}$. Estudos conduzidos no Brasil com a população infantil encontraram uma prevalência de sobrepeso/obesidade em torno de $11,9 \%$ na região Sudeste e 8,2\% no Nordeste ${ }^{21}$, sendo também esses valores variados em diversas cidades brasileiras ${ }^{22,23}$.

Nos escolares com TDC, participantes desse estudo, a maioria apresentou-se eutrófico. Porém esses achados não coincidiram com os estudos de Cairney, Hay, Faught e Hawes ${ }^{24}$, realizado no Canadá. Esses autores realizaram um estudo para verificar a relação entre TDC e sobrepeso/obesidade e constataram que o TDC pode ser um fator de risco para sobrepeso e obesidade, pois esse estado foi mais alto no grupo com TDC. Já os resultados do presente estudo podem questionar a hipótese de que o estado nutricional influencia o desempenho motor de crianças com TDC, ou pode ser que esses dados estejam relacionados às limitações do tamanho amostral. Por se tratar de um estudo novo, não existem outros estudos equivalentes que relacionem o desempenho motor e o estado nutricional de crianças com TDC, sendo difícil confrontar estes dados.

\section{CONCLUSÃO}

A presente investigação demonstrou que os escolares com TDC apresentaram desempenho inferior em todas as habilidades em relação aos escolares sem o Transtorno. Quanto ao estado nutricional, este não implicou comprometimento do desempenho motor, pois os dois grupos de escolares (com e sem TDC) apresentaram-se, na sua maioria, eutróficos. Como propostas para futuras pesquisas, recomenda-se que sejam realizados mais estudos no Brasil, tanto transversais quanto longitudinais, com o intuito de fazer levantamento de escolares com TDC e verificar o seu desempenho motor e estado nutricional, criando planos de intervenção para essa população a fim de minimizar as dificuldades motoras e oferecer um melhor desenvolvimento e qualidade de vida para esses indivíduos.

\section{REFERÊNCIAS BIBLIOGRÁFICAS}

1. Gallahue DL, Ozmun JC. Compreendendo o desenvolvimento motor: bebês, crianças, adolescentes e adultos. São Paulo: Phorte Editora, 2005.

2. Silva JAO, Dantas LE, Cattuzzo MT, Walter C, Moreira CRP, Souza CJF. Teste MABC: aplicabilidade da lista de checagem na região Sudeste do Brasil. Rev Port Cien Desp 2006;6(3):356-61.
3. Geuze RH, Jongmans MJ, Schoemaker MM, Smits-Engelsman BCM. Clinical and research diagnostic criteria or developmental coordination disorder: a review and discussion. Hum Mov Sci 2001;20(1-2):7-47.

4. Associação Americana de Psiquiatria (American Psychiatric Association - APA). Manual diagnóstico e estatístico de transtornos mentais. 4. Ed. Porto Alegre: Artmed; 2003.

5. Missiuna C. Children with Developmental Coordination Disorder: At home and in the Classroom. Ontário, Canadá: CanChild; 2003. Disponível em:<http://dcd. canchild.ca/en/> [2008 mai 30].

6. Henderson S, Sugden DA, Barnett A. Movement assessment battery for children. 2 ed. San Antonio: Harcourt Assessment; 2007.

7. Mandich AD, Polatajko HJ, Missiuna C. Developmental Coordination Disorder: Mechanisms, measurement and management. Hum Mov Sci 2003;22(4-5):407-11.

8. Cairney J, Hay JA, Mandigo J, Wade T, Faught BE, Flouris A. Developmental Coordination Disorder and reported enjoyment of physical education in children. Eur Phys Educ Rev 2007;13(1):81-98.

9. Miyahara M, Butson R, Cutfield R, Clarkson JE. A Pilot Study of Family-Focused Tele-Intervention for Children with Developmental Coordination Disorder: Development and Lessons Learned. Telemedicine and e-Health 2009;15(7):707-12.

10. França C. Desordem coordenativa desenvolvimental em crianças de 7 e 8 anos de idade. [Dissertação de Mestrado - Programa de Pós-Graduação em Ciências do Movimento Humano]. Florianópolis (SC): Universidade do Estado de Santa Catarina; 2008.

11. Souza J, Petersen RDS. Mudanças relacionadas à idade nos ajustes posturais compensatórios em crianças com e sem DCD. [Dissertação de Mestrado - Programa de Pós-Graduação em Ciências do Movimento Humano]. Porto Alegre (RS): Universidade Federal do Rio Grande do Sul; 2006

12. Secretaria de Estado da Educação de Santa Catarina Gerência de Educação da Grande Florianópolis. Brasil: 2009. Disponível em: <http://www.sed.sc.gov.br/secretaria/> [2009 mai 10].

13. Sistema de Vigilância Alimentar e Nutricional/SISVAN. Brasil: 2009; Disponível em: <http://nutricao. saude.gov.br/sisvan.php?conteudo=curvas_cresc_oms $>$ [2009 ago 20].

14. Hay JA, Hawes R, Faught BE. Evaluation of a screening instrument for developmental coordination disorder. J Adolesc Health 2004;34(4):308-13.

15. Hadders-Algra M. The neuronal group selection theory: promising principles for understanding and treating developmental motor disorders. Dev Med Child Neurol 2000;42(10):707-15.

16. Gibbs J, Appleton J, Appleton R. Dyspraxia or developmental coordination disorder? Unravelling the enigma. Arch Dis Child 2007;92(6):534-9.

17. Kadesjö B, Gillberg C. Developmental coordination disorder in Swedish 7-year-old children. J Am Acad Child Adolesc Psychiatry 1999;38(7):820-8. 
18. Wright HC, Sugden DA. The nature of Developmental Coordination Disorder: inter-and intra-group differences. Adapt Phys Act Q 1996;13(4):357-71.

19. Ruiz LM, Graupera JL, Gutiérrez M, Miyahara M. The assessment of motor coordination in children with the Movement ABC test: A comparative study among Japan, USA and Spain. Int J Appl Sport Sci 2003;15(1):22-35.

20. Ferreira AP, Oliveira CE, Franca NM. Síndrome metabólica em crianças obesas e fatores de risco para doenças cardiovasculares de acordo com a resistência à insulina (HOMA - IR). J Pediatr 2007;83(1):21-6.

21. Abrantes MM, Lamounier JA, Colosimo EA. Prevalência de sobrepeso e obesidade em crianças e adolescentes das regiões Sudeste e Nordeste. J Pediatr 2002;78(4):335-40.

22. Netto-Oliveira ER, Oliveira AAB, Nakashima ATA, Rosaneli CF, Oliveira Filho A, Rechenchosky L, et al . Sobrepeso e obesidade em crianças de diferentes níveis econômicos. Rev Bras Cineantropom Desempenho Hum 2010;12(2):83-9.
23. Farias ES, Petroski EL. Estado nutricional e atividade física de escolares da cidade de Porto Velho, RO. Rev Bras Cineantropom Desempenho Hum 2003;5(1):27-38.

24. Cairney J, Hay JA, Faught BE, Hawes R. Developmental Coordination Disorder and overweight and obesity in children aged 9-14 y 2005;Int J Obes 29(4):369-72.

\section{Endereço para correspondência}

Talita Barbosa Miranda.

Rua Deputado Antonio Edu Vieira, 1580, apto 24, Pantanal.

CEP: 88040-001 - Florianópolis, SC. Brasil. 